\title{
Significance of the astrocyte domain organization for qualitative information structuring in the brain
}

\author{
Bernhard J. Mitterauer
}

Volitronics-Institute for Basic Research, Psychopathology and Brain Philosophy, Gotthard Guenther Archives, Salzburg, Austria. Email: mitterauer@wasi.tv

Received 3 August 2010; revised 25 August 2010; accepted 27 August 2010.

\begin{abstract}
Astrocytes, the dominant glial cell type, modulate synaptic information transmission. Each astrocyte is organized in non-overlapping domains. Here, a formally based model of the possible significance of astrocyte domain organization is proposed. It is hypothesized that each astrocyte contacting $n$ neurons with $\mathbf{m}$ synapses via its processes generates dynamic domains of synaptic interactions based on qualitative criteria so that it exerts a structuring of neuronal information processing. The formalism (morphogrammatics) describes the combinatorics of the various astrocytic receptor types for occupancy with cognate neurotransmitters. Astrocytic processes are able both to contact synapses and retract from them. Rhythmic oscillations of the astrocyte may program the domain organization, where clock genes may play a role in rhythm generation. For the interpretation of a domain organization a player of a string instrument is used as a paradigm. Since astrocytes form networks (syncytia), the interactions between astrocyte domains may be comparable to the improvisations in a jazz ensemble. Given the fact of a high combinational complexity of an astrocyte domain organization, which is formally demonstrable, and an uncomputable complexity of a network of astrocyte domains, the model proposed may not be testable in biological brains, but robotics could be a real alternative.
\end{abstract}

Keywords: Astrocyte Domain Organization; Qualitative Formalism; Synaptic Information Structuring; Musical Paradigms; Robotics

\section{INTRODUCTION}

Astrocytes, the dominant glial cell type, have become the focus of much attention in the past two decades. In addition to their roles in many of the supportive functions of the brain, new functions are beginning to emerge. Abundant evidence now supports the notion that astrocytes are actively involved in synaptic transmission in most brain regions. Although astrocytes are not themselves electrically excitable, they release transmitters, triggered by increases in cytosolic $\mathrm{Ca}^{2+}$ concentrations that modulate the activity of neighboring cells, including both neurons and other glia. Astrocytes express a large number of primarily metabotropic receptors that mobilize intracellular $\mathrm{Ca}^{2+}$ stores in a phospholipase C- and inosital $(1,4,5)$ triphosphate-dependent fashion [1]. Importantly, astrocytes are thereby able to respond to neuronal activity in a receptor-dependent fashion, and in return they can modulate synaptic transmission by transmitter release, thereby permitting feedback control of neuronal activity levels [2,3]. Astrocytes release glutamate, serine and adenosine-triphosphate, and possible other transmitters that might regulate the activity of surrounding neurons [4-6]. Moreover, it is experimentally well established that astrocytes form non-overlapping territories that define functional domains [7-9]. Oberheim et al. [10] pose the question of what the functional significance of astrocyte domain organization could be, since it is as yet not fully understood. Here, I hypothesize that each astrocyte contacting $n$ neurons with $m$ synapses via its processes generates dynamic domains of synaptic interactions based on qualitative criteria, so that it exerts a structuring of neuronal information processing.

\section{OUTLINE OF AN ASTROCYTE DOMAIN ORGANIZATION}

In all mammals, protoplasmic astrocytes are organized into spatially non-overlapping domains that encompass both neurons and vasculature. An astrocyte domain defines a contiguous cohort of synapses that interacts exclusively with a single astrocyte. Synapses within a par- 
ticular territory are thereby linked via a shared astrocyte partner, independent of a neuronal networking [10]. Figure 1 shows an outline of an astrocyte domain organization. An astrocyte $\left(\mathrm{Ac}_{\mathrm{x}}\right)$ contacts the synapses (Sy) of four neurons $\left(\mathrm{N}_{1} \ldots \mathrm{N}_{4}\right)$ via its processes $\left(\mathrm{P}_{1} \ldots \mathrm{P}_{4}\right)$. Each process is equipped with one to four receptor qualities $(\mathrm{Rq})$. For example, $\mathrm{P}_{1}$ contacts the synapses of $\mathrm{N}_{2}$ exclusively via its receptors of quality a. $\mathrm{P}_{2}$ has already two receptor qualities available $(\mathrm{a}, \mathrm{b}), \mathrm{P}_{3}$ three receptor qualities $\left(\mathrm{a}, \mathrm{b}, \mathrm{c}\right.$ ) and $\mathrm{P}_{4}$ is able to contact the synapses of $\mathrm{N}_{1}$ via four receptor qualities (a, b, c, d). Astrocyte $\left(A c_{x}\right)$ is interconnected with another astrocyte $\left(A c_{y}\right)$ via gap junctions (g.j.) forming an astrocytic network (syncy- tium). The neurons per se are also interconnected (neuronal network).

It is experimentally verified that astrocytes can express almost all receptors for important transmitter systems [11]. In certain cases, individual astroglial cells express as many as five different receptor systems linked to $\mathrm{Ca}^{2+}$ mobilization [12]. Each astrocyte territory represents an island made up of many thousands of synapses (about 140.000 in the hippocampal region of the brain, for instance), whose activity is controlled by that astrocyte [9]. On the average, human astrocytes extend 40 large processes radially and symmetrically in all directions from the soma so that each astrocyte supports and modulates the function of roughly two million synapses in the cerebral cortex [10]. Astrocytic receptors are mainly located on the endfeet of the processes. Here, we apparently deal with a high combinational complexity of astrocyte-synaptic interactions.

\section{FORMAL STRUCTURE OF AN ASTROCYTE DOMAIN ORGANIZATION}

\subsection{General Considerations}

Guenther $[13,14]$ described living systems as individual units with a new universal theory of structure, called morphogrammatics. Accordingly, a theory of structure should be universal and composed of empty places. Such places can either be of equal or different quality. They can also stay empty or be occupied by anything. Based on the principle of identity and difference, these places or their structure can be analysed on three levels of complexity:

1) Protostructure: How many different places are there? This corresponds to cardinality.

2) Deuterostructure: How are these places distributed? This corresponds to distribution.

3) Tritostructure: Where are the individual places located? This corresponds to position.

Since the tritostructure represents the highest com-

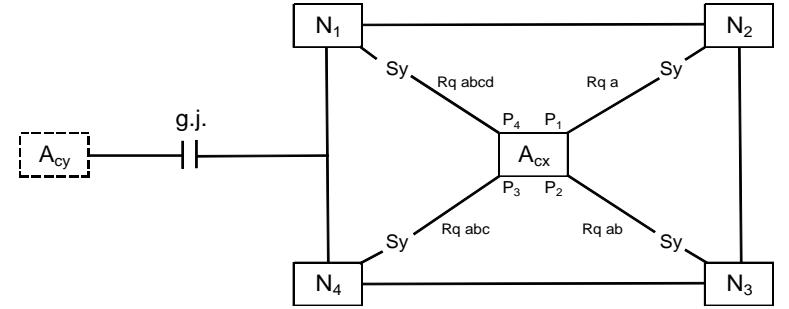

Figure 1. Outline of an astrocyte domain organization. An astrocyte $\left(\mathrm{Ac}_{\mathrm{x}}\right)$ is interconnected via four processes $\left(\mathrm{P}_{1} \ldots \mathrm{P}_{4}\right)$ with the synapses (Sy) of four neurons $\left(\mathrm{N}_{1} \ldots \mathrm{N}_{4}\right)$. Each process is on its endfoot equipped with receptors for the occupancy with neurotransmitters according to a combinational rule (shown in Table 1). As an example, the receptor $\mathrm{P}_{1}$ contacting $\mathrm{N}_{2}$ embodies only one receptor quality (Rqa). $\mathrm{P}_{2}$ contacts $\mathrm{N}_{3}$ with two different receptor qualities (Rqab). $\mathrm{P}_{3}$ contacts $\mathrm{N}_{4}$ with Rqabc and $\mathrm{P}_{4}$ contacts $\mathrm{N}_{1}$ with Rqabcd. This simple diagram represents an astrocyte domain. Astrocyte $\left(A c_{x}\right)$ is interconnected with $\mathrm{Ac}_{\mathrm{y}}$ via gap junctions (g.j.) as shown in more detail in Figure 3.

plexity, it may underly the astrocytic domain organization. Here, the morphograms are termed tritograms.

\subsection{Development of a Tritostructure Formalizing an Astrocyte Domain Organization}

Figure 2 shows the development of tritograms with $n$ places. The structure for tritograms with length 1 to 5 (5 levels) is represented by a tree. This is the generation rule: a tritogram $x$ with length $n+1$ may be generated from a tritogram $\mathrm{y}$ with length $\mathrm{n}$ if $\mathrm{x}$ is equal to $\mathrm{y}$ on the first $n$ places, e.g. 12133 may be generated from 1213 but not from 1212. The numerals are representations of domains (properties, categories) that should be viewed as 'place-holders' reserved for domains, e.g. 12133 should be read as five places for five entities, such that the first and the third entity belong to domain one, the second entity to domain two, and the fourth and fith entity to domain three.

Now let us interpret the tritostructure $(n=4)$ as an astrocyte domain organization. Table 1 shows 15 tritograms each consisting of the same or different places symbolized as numerals 1 to 4 . Since the position of the places is relevant, one can also speak of a qualitative counting of different domains [15]. This tritostructure is interpreted as the formal basis of an astrocyte with 15 processes, each embodying a receptor sheet of identical or different qualitative domains for synaptic information processing. These various receptor domains are located on the endfeet of the astrocytic processes contacting cognate neuronal synapses and modulating neurotransmission. Most importantly, it is experimentally verified that astrocytes display elaborate process extension and 


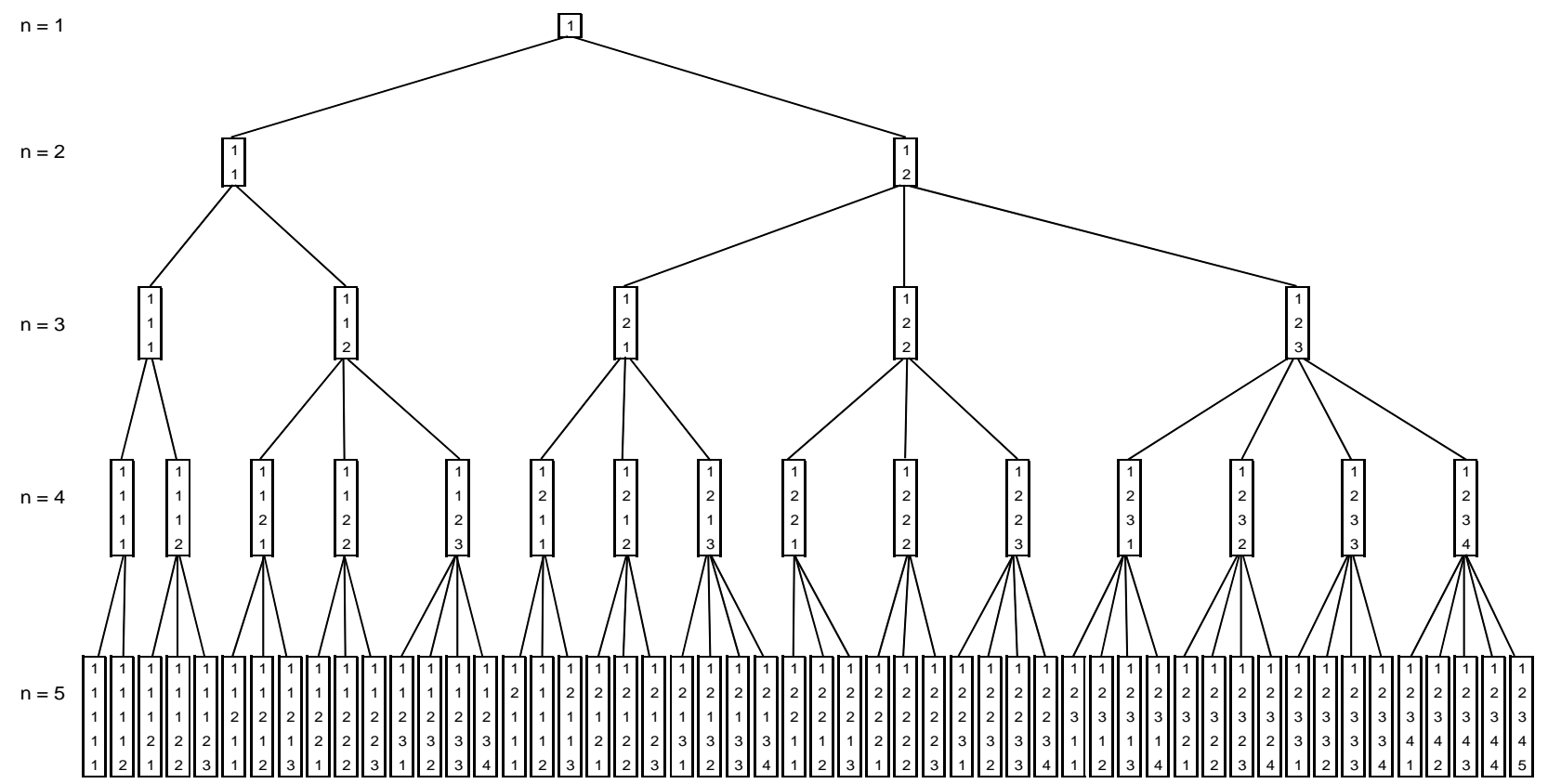

Figure 2. Tritogrammatic tree. Generation of 52 tritograms $(n=5)$ corresponding to 52 astrocytic processes. Each tritogram represents a qualitative astrocytic receptor sheet. The structure for tritograms with length 1 to 5 is represented by a tree. Generation rule: a tritogram $\mathrm{x}$ with length $\mathrm{n}+1$ may be generated from a tritogram $\mathrm{y}$ with length $\mathrm{n}$ if $\mathrm{x}$ is equal to $\mathrm{y}$ on the first $\mathrm{n}$ places, e.g. 12133 may be generated from 1213 but not from 1212. The numerals are representations of places of the same or different qualities interpreted as astrocytic receptors on the endfeet of the processes. Each tritogram corresponds to an astrocytic processor.

Table 1. Tritostructure. Generation of 15 tritograms corresponding to 15 astrocytic processes.

\begin{tabular}{ccccccccccccccccc}
\hline \multicolumn{11}{c}{ receptor qualities } \\
\hline & 1 & 1 & 1 & 1 & 1 & 1 & 1 & 1 & 1 & 1 & 1 & 1 & 1 & 1 & 1 \\
astrocytic & 1 & 1 & 1 & 1 & 1 & 2 & 2 & 2 & 2 & 2 & 2 & 2 & 2 & 2 & 2 \\
processes & 1 & 1 & 2 & 2 & 2 & 1 & 1 & 1 & 2 & 2 & 2 & 3 & 3 & 3 & 3 \\
& 1 & 2 & 1 & 2 & 3 & 1 & 2 & 3 & 1 & 2 & 3 & 1 & 2 & 3 & 4 \\
tritograms & {$[1]$} & {$[2]$} & {$[3]$} & {$[4]$} & {$[5]$} & {$[6]$} & {$[7]$} & {$[8]$} & {$[9]$} & {$[10]$} & {$[11]$} & {$[12]$} & {$[13]$} & {$[14]$} & {$[15]$} \\
\hline
\end{tabular}

15 tritograms $(n=4)$ are generated according to the generation rule shown in Figure 2. Each tritogram consists of the same or different places symbolized as numerals (1...4). Since the position is relevant one can also speak of qualitative counting of different domains. This tritostructure is interpreted as the formal basis of an astrocyte with 15 processes, each embodying a receptor sheet of identical or different qualitative domains for synaptic information processing.

retraction, and likely use the active cytoskeleton for motility $[16,17]$. To integrate these experimental results into the model proposed here, astrocytes may be searching for synapses that are equipped with neurotransmitter types appropriate for the occupancy of specific astrocytic receptors in various compositions (Table 1). Moreover, this implies that in the whole astrocyte domain not all processes or receptors are active, leading to 'breaks' in glial-neuronal synaptic interactions. Hence, we deal with a dynamic exchange that occurs between astrocytes and synapses in the sense of concerted structural plasticity of glial-neuronal interaction. However, in this context we are faced with the issue how and where such motile behavior of astrocytes may be controlled?

\section{RHYTHMIC ASTROCYTE OSCILLATIONS MAY PROGRAM THE DOMAIN ORGANIZATION}

For understanding the domain organization of an astrocyte, its rhythmic contraction waves may be decisive. Astrocytes, when they get swollen and/or depolarized, can potentially release accumulated $\mathrm{K}^{+}$, neurotransmitters, neuromodulators (e.g. taurine), and water into interstitial fluid in a pulsatile manner [18]. Such discharge processes represent mechanisms by which astrocyte networks (syncytia) could influence neuronal firing in a 
coordinated fashion [19]. Moreover, astrocytes may play a direct role in generating pacemaker rhythms [20].

This originally speculative assumption has already been verified. Parri et al. [21] showed that astrocytes in situ could act as a primary source for generating neuronal activity in the mammalian central nervous system. Slow astrocyte calcium oscillations (every 5 to 6 minutes) occur spontaneously (without prior neuronal activation) and can cause excitations in nearby neurons. Considering experimental findings of the structural interplay between astrocytes and synapses in hippocampal slices, dynamic structural changes in astrocytes help control the degree of glial-neuronal communication [17]. Since the time scales of both astrocyte calcium oscillations and morphological changes in astrocytes occur within minutes, a pacemaker function may determine the motility of astrocyte processes and the generation of a structural pattern of astrocyte-synaptic interactions.

In comparison of the rapid synaptic information processing within milliseconds, the pulsations and morphological changes of astrocytes are relatively slow. Thus, it is often argued that glia cannot exert an effect in synaptic information processing. This argument may be erroneous if cognitive processes are considered. Cognitive processes, such as thinking and planning, etc., occur in a timescale of minutes, hours, days or weeks, since they need a relatively long timespan. I hypothesize that an astrocyte domain is organized within this long time scale generating a specific qualitative structure of glial-neuronal information processing.

\section{PLAYER OF A STRING INSTRUMENT AS A PARADIGM}

For a general understanding of a single astrocyte domain organization, I will use a player of a string instrument as a paradigm. Let us assume he/she has a melody in mind. At the same time he/she would like to know what the melody sounds like. He/she takes his/her instrument and presses the fingers of the left hand on the strings over precisely those places that will produce the desired melody. In parallel, the fingers of the right hand must activate the strings. Now, the formalism of tritograms (Table 1) allows the following interpretation:

Supposing the instrument is equipped with four strings, each embodies an empty place of a specific quality comparable to four types of neuronal synapses. According to the underlying combinatorics, the same and different strings, if activated, can be chosen by pressing them. This means in biological terms that the astrocyte (player) contacts synapses (strings) via its processes (fingers) generating a specific structure of astrocyte-synaptic interactions according to a combination rule in the sense of an astrocyte domain organiza- tion (melody). Most importantly, not all strings are always pressed in generating a melody, but often single strings must be selected. Here, we may deal with the same dynamic organization principle as in an astrocyte domain where processes both contact synapses and retract from them as well.

In attempting to interpret the organization of astrocyte domains into networks (syncytia), the musical paradigm can be elaborated further, as shown by the harmonization in a jazz ensemble.

\section{NETWORK ORGANIZATION OF ASTROCYTE DOMAINS COMPARABLE TO HARMONIZATION IN A JAZZ ENSEMBLE}

First, the main biological structure of an astrocytic network, called syncytium, must be outlined.

\subsection{Outline of an Astrocytic Syncytium}

Figure 3 shows a diagrammatic schema depicting an astrocytic syncytium composed of two astrocytes $\left(\mathrm{Ac}_{1}\right.$, $\mathrm{Ac}_{2}$ ) interconnected via gap junctions (g.j.). Each astrocyte contacts four synapses (Sy) with four different qualities (a, b, c, d) building an astrocyte-neuronal domain. A quality (astrocytic receptor domain) is defined as the specific neurotranmsitter type that operates in synaptic neurotransmission, say glutamate (a), GABA (b), noradrenaline (c), dopamine (d). The gap junctions consist of four identified astrocytic connexins Cx 43, Cx 30, Cx 26, and Cx 45, forming homotypic (same connexins) and heterotypic (different connexins) gap junction channels (for the sake of clarity not shown in the figure). As already discussed, the interactions of astrocytes with synapses occur in a pulsatile manner in vari-

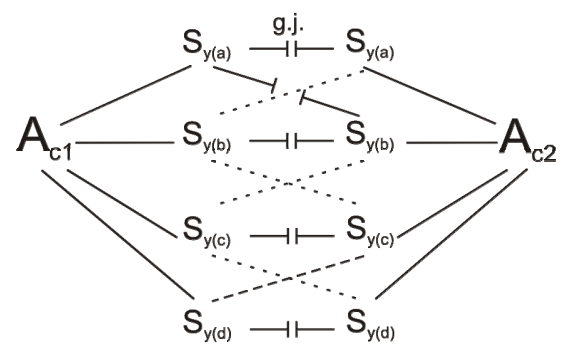

Figure 3. Diagrammatic schema of an astrocytic syncytium. Two astrocytes $\left(\mathrm{Ac}_{1}, \mathrm{Ac}_{2}\right)$ are interconnected via gap junctions (g.j.). Each astrocyte contacts four synapses (Sy) with four different qualities (a, b, c, d) building two astrocytic-neuronal domains. Since these two domains are interconnected via gap junctions, a network is generated, called syncytium. 
ous time scales. Although astrocyte-synaptic interactions within milliseconds are also identified [22], the dominating time scales are minutes to hours. Moreover, do the organizational principles of a single astrocyte domain also hold in an astrocytic syncytium? Can they also be compared to the creation of music? How do the individual astrocyte domains (instruments) with different time scales cooperate to produce an integrative behavior? Let us take the improvisation in a jazz ensemble as an example.

\subsection{Harmonization in a Jazz Ensemble}

Members of a classical orchestra have a strictly defined environment as they perform directly from notes of the page. The jazz ensemble, however, plays within a defined harmonic structure in which every musician can improvise, develop, and vary themes. Certain themes can be played best by one instrument while the other instruments play accompaniment or rest until their turn to improvise. Every instrument can principally carry the melody. Each musician listens to what the others play in that moment and participates in the harmonization or rests. Each musician can in his way synchronize his musical or harmonic intention with the 'environment' of the rest of the ensemble by playing with them, by improvising a solo, or by resting.

A comparable organization may be at work in the astrocytic syncytium and its synaptic interactions with the neuronal system. My biological interpretation is this: each astrocyte domain stands for a musician playing the same or different intstruments. They are able to listen to one another, since astrocytes are interconnected via gap junctions. Astrocyte domains can rest by retracting their processes from synapses or by not activating (playing) a synapse, when it is silent. Silent synapses are experimentally well established [23]. Importantly, here we do not deal with synchronization, but with a special kind of self-organization of intentional and environment-dependent structuring of information, comparable to harmonic structuring in music [24,25].

\section{CLOCK GENES COULD BE RESPONSIBLE FOR THE RHYTHMIC OPERATIONS OF ASTROCYTE DOMAINS}

Molecular and cellular processes in the brain are determined by a plethora of various biological clocks. Not only do circadian rhythms exist, but also ultradian rhythms [26]. These ultradian rhythms include a time scale from pico-seconds and milliseconds to minutes and hours [27]. The investigation of circadian clocks is making significant progress. The McKnight group reported that the transcription factor neuronal PAS domain protein 2 (NPAS 2) likely functions as part of a molecular clock operative in the mammalian forebrain [28]. The discovery may provide a molecular link between circadian oscillations and energy homeostasis interlocked through negative feedback loops [29]. The problem of the synchronization of this rhythmic diversity, however, remains unsolved. The following hypothesis may resolve this issue: a domain or domains whose rhythm at a certain moment best corresponds to internal and external environment conditions command, and therefore, determine the rhythm of the molecular processes of the entire system. This is comparable to a melody which can be best realized by certain instrument groups while other instrument groups assume a secondary function, as accompaniment or rest altogether. However, since theoretically all instruments could be melodic carriers, a "redundancy of potential command" [30] rules orchestration. Those instruments best suited through their timbre, tone color or intensity of sound in any given "environmental situation” will take a predominant role. Concerning the interaction of biological clocks, Lloyd [27] proposes a safety-net of redundancy and checkpoints, so that if one timing circuit fails another deputizes. However, Lloyd does not penetrate the principle of the redundancy of potential command. At least in the brains of Drosophila melanogaster neuronal and glial cells containing clock genes have been identified [31]. A recent study focuses on glial cells that may be responsible for setting the beat [32]. This experimental finding could implicate that the rhythmic pulsations of an astrocyte could be generated and controlled by clock genes. Presently, one can only guess that this may also be the case in human brains, since pertinent data are not yet available.

\section{FUTURE PROSPECTS}

The model of a single astrocyte domain organization and the generation of networks operate on qualitative criteria what the interaction of the astrocytic receptors with the neuronal compartments of a synapse concerns. This interaction is highly dynamic and occurs in a pulsatile manner. Since only a specific amount of astrocyte processes contact cognate synapses at a given moment, astrocytes may exert an information structuring function comparable to playing a music instrument or improvising in a jazz ensemble. Moreover, this theoretical model should be testable.

Given the estimation that the mean number of astrocytic processes is 40 with a potential of contacting of about two million synapses, testing my model in biological brains may seem to be impossible. However, a real alternative could be a computer simulation of the dynamic organization of an astrocyte based on the pro- 
posed formalism. As a first technical step in the implementation of a glial-neuronal domain, I have simulated a “clocked perception system” [33]. Since then, the model has been further developed, especially what a possible generation of intentional programs in the astrocytic syncytium concerns [34]. If we could build a chip it might function comparable to an astrocyte domain interacting with n-neuronal synapses. Moreover, if a set of chips interact like astrocytes in their syncytium, a robot brain that operates according to qualitative criteria structuring environmental information could stepwise be constructed. Remarkably, although neuronal computing or neuronal network technologies are dominating, recently computer modelling of glial-neuronal interactions in synapses is emerging [35].

\section{REFERENCES}

[1] Volterra, A. and Meldolesi, J. (2005) Astrocytes, from brain glue to communication elements: The revolution continues. Nature Reviews Neuroscience, 6, 626-640.

[2] Auld, D.S. and Robitaille, R. (2003) Glial cells and neurotransmission: An inclusive view of synaptic function. Neuron, 40, 389-400.

[3] Halassa, M.M. and Haydon, P.G. (2010) Integrated brain circuits: Astrocytic networks modulate neuronal activity and behaviour. Annual Review of Physiology, 72, 335355.

[4] Haydon, P.G. (2001) Glia: Listening and talking to the synapse. Nature Reviews Neuroscience, 2, 185-193.

[5] Haydon, P.G. and Carmignoto, G. (2006) Astrocyte control of synaptic transmission and neurovascular coupling. Physiological Review, 86, 1009-1031.

[6] Verkhratsky, A.and Butt, A. (2007) Glial neurobiology. Wiley, West Sussex.

[7] Nedergaard, M., Ransom, B. and Goldman, S.A. (2003) New roles for astrocytes: Redefining the functional architecture of the brain. Trends in Neuroscience, 25, 523530.

[8] Halassa, M.M., Fellin, T., Takano, H., Dong, J. and Haydon, P.G. (2007) Synaptic islands defined by the territory of a single astrocyte. The Journal of Neuroscience, 27, 6473-6477.

[9] Santello, M. and Volterra, A. (2010) Astrocytes as aidemémoires. Nature, 463, 169-170.

[10] Oberheim, N.A., Wang, X., Goldman, S. and Nedergaard, M. (2006) Astrocytic complexity distinguishes the human brain. Trends in Neuroscience, 29, 547-553.

[11] Kettenmann, H. and Steinhäuser, C. (2005) Receptors for neurotransmitters and hormones. In: Kettenmann, $\mathrm{H}$. and Ransom, B.R., Eds., Neuroglia, Oxford University Press, Oxford, 131-145.

[12] McCarthy, K.D. and Salm, A.K. (1991) Pharmacologically- distinct subsets of astroglia can be identified by their calcium response to neuroligands. Neuroscience, 2-3, 325-333.

[13] Guenther, G. (1962) Cybernetic ontology and transjunctional operations. In: Yovits, M.C., Jacobi, G.T. and
Goldstein, G.D., Eds., Self-organizing Systems, Spartan Books, Washington DC, 313-392.

[14] Guenther, G. (1976) Beiträge zur Grundlegung einer operations fähigen Dialektik. Band I, Meiner, Hamburg.

[15] Thomas, G.G. (1985) Introduction to kenogrammatics. Proceedings of the 13th Winter School on Abstract Analysis, section of Topology, Rendiconti del Circolo Matematico di Palermo, Supplemento No. 11, serie II, , 113-123.

[16] Hirrlinger, J., Hulsmann, S. and Kirchhoff, F. (2004) Astroglial processes show spontaneous motility at active synaptic terminals in situ. European Journal of Neuroscience, 20, 2235-2239.

[17] Haber, M., Zhou, L. and Murai, K.K. (2006) Cooperative astrocyte and dendritic spine dynamics at hippocampal excitatory synapses. Journal of Neuroscience, 26, 88878891.

[18] Cooper, M.S. (1995) Intercellular signalling in neuronal-glial networks. BioSystems, 34, 65-85.

[19] Newman, E.A. and Zahs, K.R. (1997) Calcium waves in retinal glial cells. Science, 275, 844-846.

[20] Mitterauer, B., Garvin, A.M. and Dirnhofer, R. (2000) The sudden infant death syndrome: A neuro-molecular hypothesis. Neuroscientist, 6, 154-158.

[21] Parri, H.R., Gould, T.M. and Crunelli, V. (2001) Spontaneous astrocytic $\mathrm{Ca}^{2+}$ oscillations in situ drive NMDARmediated neuronal excitation. Nature Neuroscience, 4, 803-812.

[22] Winship, I.R., Plaa, N. and Murphy, T.H. (2007) Rapid astrocyte calcium signals correlate with neuronal activity and onset of the hemodynamic response in vivo. Journal of Neuroscience, 27, 6268-6272.

[23] Barnes, S.J. and Finnerty, G.T. (2010) Sensory experience and cortical rewiring. Neuroscientist, 16, 186-198.

[24] Mitterauer, B. (2001) Clocked perception system. Journal of Intelligent Systems, 11, 269-298.

[25] Mitterauer, B. and Kopp, C. (2003) The self-composing brain: Towards a glial-neuronal brain theory. Brain and Cognition, 51, 357-367.

[26] Iwasaki, K. and Thomas, J.H. (1997) Genetics in rhythm. Trends in genetics, 13, 111-115.

[27] Lloyd, D. (1998) Circadian and ultradian clock-controlled rhythms in unicellular microorganisms. Advances in Microbiological Physiology, 39, 291-338.

[28] Reick, M., Garcia, J.A., Dudley, C. and McKnight, S.L. (2001) NPAS 2: An analog of clock operative in the mammalian forebrain. Science, 293, 506-509.

[29] Schibler, U., Ripperger, J.A. and Brown, S.A. (2001) Chronobiology-reducing time. Science, 293, 437-438.

[30] McCulloch, W.S. (1965) Embodiments of mind. The MIT Press, Cambridge.

[31] Helfrich-Förster, C. (1995) The period clock gene is expressed in the central nervous system neurons which also produce a neuropeptide that reveals the projections of circadian pacemaker cells within the brain of Drosophila melanogaster. Proceedings of the National Academy of Sciences of USA, Vol. 92, , pp. 612-616.

[32] Flight, M.H. (2007) Circadian rhythms: glia set the beat. Nature Reviews Neuroscience, Vol. 8, , pp. 654.

[33] Mitterauer, B. (2004) Computer System, particularly for simulation of human perception via sense organs. United States Patent, 6, 697, 789B2. 
[34] Mitterauer, B. (2007) Where and how could intentional programs be generated in the brain? A hypothetical model based on glial-neuronal interactions. BioSystems, 88, 101-112.
[35] Nadkarni, S., Jung, P. and Levine, H. (2008) Astrocyte optimize the synaptic transmission of information. PloS Computational Biology, 4, 1-11. 